\title{
5-HT in the dorsal raphe nucleus is involved in the effects of 100-Hz electro-acupuncture on the pain-depression dyad in rats
}

\author{
YUAN-YUAN WU ${ }^{1}$, YONG-LIANG JIANG ${ }^{1}$, XIAO-FEN HE $^{1}$, XIAO-YUN ZHAO ${ }^{2}$, XIAO-MEI SHAO ${ }^{1}$, \\ JING SUN $^{1}$, ZUI SHEN ${ }^{1}$, SHEN-YUN SHOU ${ }^{1}$, JUN-JUN WEI ${ }^{1}$, JIA-YU YE ${ }^{1}$, SI-SI YAN ${ }^{1}$ and JIAN-QIAO FANG ${ }^{1}$ \\ ${ }^{1}$ Department of Neurobiology and Acupuncture Research, The Third Clinical Medical College, \\ Zhejiang Chinese Medical University, Hangzhou, Zhejiang 310053; ${ }^{2}$ Department of Orthopedics \\ and Traumatology, Shanxi Hospital of Traditional Chinese Medicine, Xian, Shanxi 710000, P.R. China
}

Received March 7, 2016; Accepted February 17, 2017

DOI: $10.3892 / e t m .2017 .4479$

\begin{abstract}
The pain-depression dyad is becoming widespread in the clinic and is attracting increasing attention. A previous study by our group found that $100-\mathrm{Hz}$ electro-acupuncture (EA), but not 2-, 50- and 2/100-Hz EA, was effective against the reserpine-induced pain-depression dyad. This finding is in contrast to the fact that low-frequency EA is commonly used to treat supraspinal-originating diseases. The present study aimed to investigate the mechanism underlying the effects of $100-\mathrm{Hz}$ EA on the pain-depression dyad. Repeated reserpine injection was found to induce allodynia and depressive behaviors in rats. It decreased 5-hydroxytryptamine (5-HT) levels and immunoreactive expressions in the dorsal raphe nucleus (DRN). 100-Hz EA alleviated the pain-depression dyad and upregulated 5-HT in the DRN of reserpine-injected rats. Intracerebroventricular injection of para-chlorophenylalanine, an inhibitor of 5-HT resynthesis, suppressed the upregulation of $5-\mathrm{HT}$ in the DRN by $100-\mathrm{Hz}$ EA and partially counteracted the analgesic and anti-depressive effects of $100-\mathrm{Hz}$ EA. The present study was the first to demonstrate that 5 -HT in the DRN is involved in mediating the analgesic and anti-depressive effects of 100-Hz EA on the pain-depression dyad. This
\end{abstract}

Correspondence to: Dr Jian-Qiao Fang, Department of Neurobiology and Acupuncture Research, The Third Clinical Medical College, Zhejiang Chinese Medical University, 548 Binwen Road, Hangzhou, Zhejiang 310053, P.R. China

E-mail: fangjianqiao7532@163.com

Abbreviations: 5-HT, 5-hydroxytryptamine (serotonin); DRN, dorsal raphe nucleus; EA, electro-acupuncture; PWT, paw withdrawal threshold; pCPA, para-chlorophenylalanine; OF, open field; EZM, elevated zero maze; ST 36, Zusanli, $5 \mathrm{~mm}$ lateral to the anterior tubercule of tibia; SP 6, Sanyinjiao, $10 \mathrm{~mm}$ proximal to the prominence of medial malleolus; HPLC-ECD, high-performance liquid chromatography and electrochemistry detection; TBST, Tris-buffered saline and Tween-20

Key words: pain-depression dyad, electro-acupuncture, 5-hydroxytryptamine, dorsal raphe nucleus, rat finding provided a scientific basis for high-frequency EA as a potential treatment for the pain-depression dyad.

\section{Introduction}

The pain-depression dyad is characterized by widespread pain, tenderness to palpation and various concomitant symptoms, including affective disorders such as depression (1). This syndrome is becoming increasingly widespread in the clinic and is attracting increasing amounts of attention (2-5). It is usually treated with antidepressants and antiepileptics. However, due to heavy side effects of antidepressants, it is difficult for patients to adhere to the medication for a long duration (6). Researchers have begun to focus on non-pharmaceutical therapies such as music therapy and psychotherapy, in an attempt to identify an ideal and systemic therapy for the pain-depression dyad.

Acupuncture, particularly electro-acupuncture (EA), has been approved worldwide to be effective for pain or emotional problems (7-9). Low-frequency EA can relieve pain mainly at the supraspinal level and the spinal cord, while high-frequency EA mainly acts via the spinal cord (10). Low-frequency EA also effectively attenuates depression (11). Considering that the pain-depression dyad is associated to lesions at the supraspinal level $(1,12)$, it was hypothesized that low-frequency EA is also more effective than high-frequency EA in treating the pain-depression dyad. However, by comparing the effects of 2-, 50-, 100- and 2/100-Hz EA on the pain-depression dyad, a previous study by our group found that only 100-Hz EA effectively alleviated allodynia and depression (13). Consequently, the present study was performed to investigate the underlying mechanism of this effect of $100-\mathrm{Hz}$ EA.

Accumulating evidence has shown that the serotonergic system in the dorsal raphe nucleus (DRN) participates in the descending modulation of pain $(14,15)$. It also has an important role in the induction and manipulation of emotional disorders $(16,17)$. Studies have shown that the serotonergic system in DRN has a role in the effects of EA on pain or emotional disorders $(18,19)$. Whether the serotonergic system in DRN is also involved in the effects of $100-\mathrm{Hz}$ EA on the pain-depression dyad has remained elusive, which was therefore the focus of the present study. 


\section{Materials and methods}

Animals. A total of 50 male Sprague-Dawley rats (weight, $250 \pm 20 \mathrm{~g}$; age, 8 weeks old) were purchased from the Shanghai Laboratory Animal Center (Shanghai, China) and housed in $40 \times 50 \times 25 \mathrm{~cm}$ cages at room temperature $\left(25 \pm 1^{\circ} \mathrm{C}\right)$ with ad libitum access to food and water. The animals were housed in groups of 5-6 rats with a 12-h light/dark cycle. All animal experiments were performed in accordance with the regulations of the State Science and Technology Commission for the Care and Use of Laboratory Animals (no. 2, 1988). The present study was approved by the Ethics Committee of Zhejiang Chinese Medical University (Hangzhou, China).

Experimental design. All animals were implanted with a guide cannula and allowed to recover for seven days. The animals were then randomly assigned to a normal group or a reserpine treatment group. The reserpine treatment group included the following subgroups: Model group, EA group and pCPA + EA group. Mechanical allodynia was assessed via paw withdrawal threshold (PWT) to a mechanical stimulus. The reserpine solution was subcutaneously injected once daily for three consecutive days [day(d)1-d3]. The PWT was assessed 1 day after the last reserpine injection (d4). Intracerebroventricular (i.c.v.) injection of a para-chlorophenylalanine (pCPA) or sterile saline was administrated immediately after the PWT tests. Then EA treatment was applied on the same day. Another PWT test was performed $30 \mathrm{~min}$ after EA treatment. On d5, the micro-injection and EA treatment were repeated. PWT, open field (OF) and elevated zero maze (EZM) tests were performed in sequence $30 \mathrm{~min}$ after EA treatment. All rats were immediately sacrificed after the behavioral tests to obtain DRN samples (Fig. 1).

Guide cannula implantation. The surgery was performed as described previously (20), with slight modifications. On the day of surgery, the animals were anesthetized with $0.5 \mathrm{ml} / \mathrm{kg}$ of 7\% chloral hydrate (Sinopharm Chemical Reagent Co., Ltd, Shanghai, China). Each rat was then placed in a stereotaxic apparatus (68002; RWD Life Science Co., Ltd, Shenzhen, China). A small incision was made to expose the skull and a burr hole was drilled. And i.c.v. guide cannula (62001; RWD Life Science Co., Ltd) was implanted according to the following coordinates, which were based on a standard rat brain stereotaxic atlas (21): $0.9 \mathrm{~mm}$ posterior to the bregma, $1.5 \mathrm{~mm}$ lateral to the midline and $3.8 \mathrm{~mm}$ ventral from the surface of the skull. The guide cannula was affixed to the skull using two stainless steel screws and dental cement. All of the animals were allowed to recover for seven days after surgery.

Induction of the pain-depression dyad. Reserpine powder (100 mg; Sigma-Aldrich, Merck KGaA, Darmstadt, Germany) was dissolved in $400 \mu \mathrm{l}$ glacial acetic acid and then diluted with distilled water to $20 \mathrm{ml}$. The animals in the reserpine treatment group were injected subcutaneously with a reserpine solution $(0.2 \mathrm{ml} / \mathrm{kg}$ daily) for three consecutive days as previously described (13). The rats in the normal group were injected subcutaneously with a vehicle solution ( $2 \%$ solution of glacial acetic acid in distilled water).
Micro-injection of $p C P A$. In the pCPA + EA group, pCPA (20 mg/ml; Sigma-Aldrich; Merck KGaA), an inhibitor of serotonin (5-hydroxytryptamine; 5-HT) resynthesis, was injected directly into the lateral ventricle. An injection cannula was connected to a $250-\mu 1$ Hamilton syringe with polyethylene tubing [outer diameter, $0.85 \mathrm{~mm}$; inner diameter (ID), $0.42 \mathrm{~mm}$; RWD Life Science Co., Ltd] and back-filled with the pCPA solution. The injection cannula was inserted into the guide cannula and $10 \mu \mathrm{l}$ of the pCPA solution was injected (i.c.v.) using a microsyringe infusion pump (UMP3; World Precision Instruments Inc., Sarasota, FL, USA) at a rate of $1 \mu \mathrm{l} / \mathrm{min}$. The injection cannula was kept in the guide cannula for $10 \mathrm{~min}$ after injection. The rats in other groups were injected with $10 \mu \mathrm{l}$ sterile saline.

EA treatment. The bilateral Zusanli (ST 36, $5 \mathrm{~mm}$ lateral to the anterior tubercule of the tibia) and Sanyinjiao (SP 6, $10 \mathrm{~mm}$ proximal to the prominence of medial malleolus) acupoints were selected as in the previous study by our group (13). Stainless steel acupuncture needles $(0.25 \mathrm{~mm}$ in diameter, $13 \mathrm{~mm}$ in length) were inserted into the acupoints at a depth of $5 \mathrm{~mm}$. The two ipsilateral needles were connected to the output terminals of a Han's Acupoint Nerve Stimulator (LH-202H; Huawei Co. Ltd., Beijing, China). The EA parameters were adopted as follows: Square wave current output (pulse width, $0.2 \mathrm{msec}$ ); stimulation intensities of $1.0,1.5$ and $2.0 \mathrm{~mA}$, each for $15 \mathrm{~min}$ in sequence; stimulation frequency of $100 \mathrm{~Hz}$. Animals were awake and calmed by placing their heads in black hoods with no physical restraint during EA treatment. EA was performed on $\mathrm{d} 4$ and $\mathrm{d} 5$.

Assessment of mechanical allodynia. Mechanical allodynia was measured using an electronic von Frey instrument (EVF-3; Bioseb In Vivo Research Instruments, Chaville, France) $(22,23)$. Rats were placed on an elevated metal mesh floor and allowed to adapt for $15 \mathrm{~min}$. The stimulus was applied to the left hind paw for $5 \mathrm{sec}$. The plastic rod was pushed against the left hind paw with linear ascending force until a robust and immediate withdrawal occurred. The PWT was calculated as the mean of three tests with intervals of $30 \mathrm{sec}$.

Behavioral tests of depression and associated emotional disorders. Emotional behavior was quantified using the $\mathrm{OF}$ test and EZM test, which are generally used to evaluate depression and anxiety $(24,25)$. All exterior lights were blocked and the ambient noise in the testing room was maintained below $40 \mathrm{db}$; abrupt loud noises that may have altered locomotion or produce prolonged immobility were avoided during testing. The room temperature was maintained at $\sim 25^{\circ} \mathrm{C}$.

The OF test was performed as follows (13): Four square $\mathrm{OF}$ arenas $(100 \mathrm{~cm}$ in diameter, $100 \mathrm{~cm}$ in width and $100 \mathrm{~cm}$ in height) constructed with black plexiglass were placed together to form the apparatus. The entire apparatus was wiped with $75 \%$ ethanol prior to each trial. Animals were placed in the testing room $1 \mathrm{~h}$ before the test. Each animal was placed in the center of the arena for $20 \mathrm{sec}$ at the beginning of the trial to adapt to the environment and the behavior was then videotaped for $5 \mathrm{~min}$ and quantified by the SMART 3.0 system (Panlab Harvard Apparatus, Barcelona, Spain). 


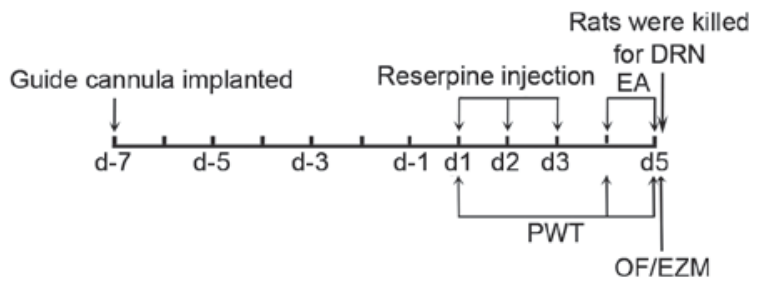

Figure 1.Experimental procedure. All animals were implanted with a cannula (d-6) and allowed to recover for seven days. Mechanical allodynia was assessed via PWT (d1). Reserpine solution was subcutaneously injected once daily for three consecutive days (d1-d3). PWT was assessed prior to and after EA treatment (d4). On d5, EA and PWT assessment were repeated, followed by the OF and EZM tests in sequence. All rats were sacrificed in order to obtain dorsal raphe nucleus samples. PWT, paw withdrawal threshold; EA, electroacupuncture; d, day; EZM, elevated zero maze; OF, open field.

The EZM test was performed as follows (13): A maze with a black metallic annular platform $(100 \mathrm{~cm}$ in diameter, $25 \mathrm{~cm}$ in width and $55 \mathrm{~cm}$ in height) was equally divided into four quadrants. Two opposite quadrants (closed arms) were enclosed by black metallic walls (30 cm in height) on the inner and outer edges of the platform, while the remaining two opposite quadrants (open arms) remained uncovered (24). The animals were placed in the testing room $1 \mathrm{~h}$ prior to the test. The entire apparatus was wiped with $75 \%$ ethanol prior to each trial. The animal was placed in the center of a closed arm for $20 \mathrm{sec}$ to adapt to the environment and its behavior was then videotaped for $5 \mathrm{~min}$ and quantified by the SMART 3.0 system.

Detection of 5-HT levels by high-performance liquid chromatography and electrochemistry detection (HPLC-ECD). HPLC-ECD was performed as described previously (26), with slight modifications. A stock solution of 5-HT (H9523; Sigma-Aldrich; Merck KGaA) at a concentration of $1 \mu \mathrm{g} / \mathrm{ml}$ was prepared as a standard. 5-HT powder was added to $0.1 \mathrm{~mol} / 1$ hydrochloric acid, which was then dissolved in $0.1 \mathrm{~mol} / 1$ perchloric acid including $1 \mathrm{~mol} / 1$ EDTA (Sigma-Aldrich; Merck KGaA). The working standard solutions were prepared by serially diluting the stock solutions to concentrations of $160,80,40,20,10,5$ and $2.5 \mathrm{ng} / \mathrm{ml}$. All solutions were filtered through a $0.22-\mu \mathrm{m}$ Millipore filter prior to injection into the HPLC-ECD system (Agilent 1200 HPLC system; Agilent Technologies, Inc., Santa Clara, CA, USA) equipped with an ESA Coulochem III detector (Thermo Fisher Scientific, Inc., Waltham, MA, USA). Standard solutions (10 $\mu \mathrm{l})$ were injected using an autosampler to generate a standard curve by HPLC-ECD analysis.

Samples were prepared in accordance with the procedure of Pani et al (27). The animals were anesthetized and transcardially perfused with ice-cold saline to remove circulating blood. The brains were quickly removed from the calvaria and placed in a cooled rat brain matrix (68711; RWD Life Science Co., Ltd). The DRN was dissected (21), weighed and stored at $-80^{\circ} \mathrm{C}$. The samples were ultrasonically homogenized in $0.1 \mathrm{~mol} / 1$ perchloric acid containing $1 \mathrm{~mol} / 1$ EDTA $(10 \mu 1$ solution for each milligram of tissue). The homogenate was centrifuged at $25,300 \mathrm{x} \mathrm{g}$ at $4^{\circ} \mathrm{C}$ for $15 \mathrm{~min}$. The supernatant was filtered through a $0.22-\mu \mathrm{m}$ millipore filter and stored at $-80^{\circ} \mathrm{C}$.

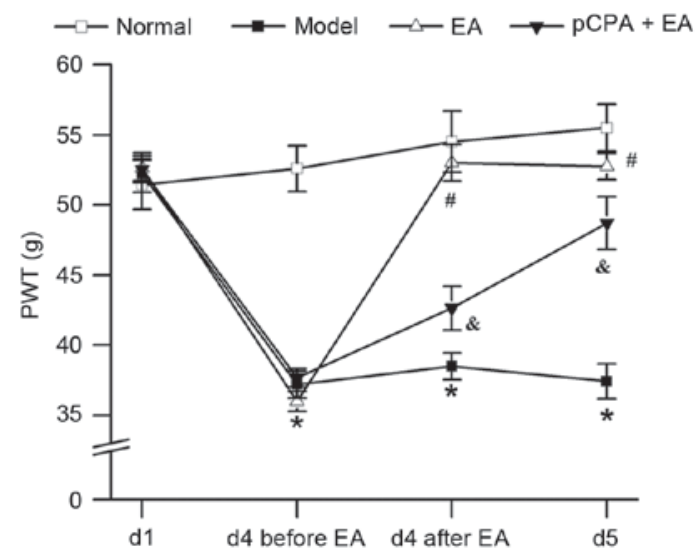

Figure 2. Effects of EA on the PWT in rats with pain-depression dyad. The PWT was measured with an electronic von Frey apparatus at d1, d4 before EA, d4 after EA and d5. Values are expressed as the mean \pm standard error of the mean $(n=10)$. ${ }^{*} \mathrm{P}<0.05$, vs. normal group; ${ }^{\#} \mathrm{P}<0.05$, vs. model group; ${ }^{\&} \mathrm{P}<0.05$, vs. EA group. PWT, paw withdrawal threshold; d, day; EA electroacupuncture; pCPA, para-chlorophenylalanine.

The following working conditions were maintained in the HPLC-ECD system for the detection of 5-HT: Gradient elution; mobile phase: $75 \mathrm{mmol} / 1 \mathrm{NaH}_{2} \mathrm{PO}_{4} \cdot \mathrm{H}_{2} \mathrm{O} ; 1.7 \mathrm{mmol} / \mathrm{l}$ sodium octane sulfonate; $100 \mu \mathrm{l} / 1$ triethyl amine; $25 \mathrm{mmol} / \mathrm{l}$ EDTA; $10 \%$ acetonitrile; $\mathrm{pH}$ 3.0; C18 reversed-phase column (inner diameter, $3.2 \mathrm{~mm}$; length, $150 \mathrm{~mm}$; MD-150 ODS; Thermo fisher Scientific, Inc.); flow rate, $0.6 \mathrm{ml} / \mathrm{min}$; temperature, $33^{\circ} \mathrm{C}$; injection volume, $10 \mu \mathrm{l}$; detector Model, 5014B (analytical cell) and 5020 (guard cell); cell potentials $\mathrm{E}_{1}, \mathrm{E}_{2}$ and $\mathrm{E}_{\mathrm{GC}}:-150,+220$ and $+270 \mathrm{mV}$, respectively; full scale/range, $100 \mathrm{nA}$; signal output voltage, $1.0 \mathrm{~V}$. The 5-HT levels are expressed as ng/g of wet tissue.

Immunofluorescence of 5-HT expression in the DRN. After the behavioral tests, the animals were anesthetized with chloral hydrate $(3.5 \mathrm{mg} / \mathrm{kg}$, intraperitoneal injection) and transcardially perfused with $150 \mathrm{ml}$ pre-cooled saline followed by $400 \mathrm{ml}$ of a $4 \%$ paraformaldehyde solution. The brains were removed and post-fixed in paraformaldehyde for $24 \mathrm{~h}$ prior to being placed in a $15 \%$ sucrose solution overnight. The brains were transferred to a $30 \%$ sucrose solution and incubated for $72 \mathrm{~h}$ prior to embedding in optimal cutting temperature matrix. Cryostat sections were cut at $30 \mu \mathrm{m}$ around the DRN region (bregma $-7.50,8.00$ and $8.50 \mathrm{~mm}$ ) on a sliding microtome and blocked in 5\% donkey serum (ab7475; Abcam, Cambridge, UK) in Tris-buffered saline containing Tween-20 (TBST)/Triton for $60 \mathrm{~min}$. The sections were then incubated at $4^{\circ} \mathrm{C}$ overnight in TBST containing an anti-5-HT primary antibody (1:100 dilution; cat. no. ab10385; Abcam). Immunoreactivity to the antigen was visualized using an Alexa Fluor 488-conjugated secondary antibody (1:1,000 dilution; cat. no. 103715; Jackson ImmunoResearch Laboratories, Inc., Bar Harbor, ME, USA). Images were obtained using a fluorescence microscope (Olympus IX71; Olympus, Tokyo, Japan) equipped with Image-Pro Insight 8.0 software (Media Cybernetics, Rockville, MD, USA).

Statistical analysis. Values are expressed as the mean \pm standard error of the mean. SPSS 16.0 software (SPSS, Inc., 
A
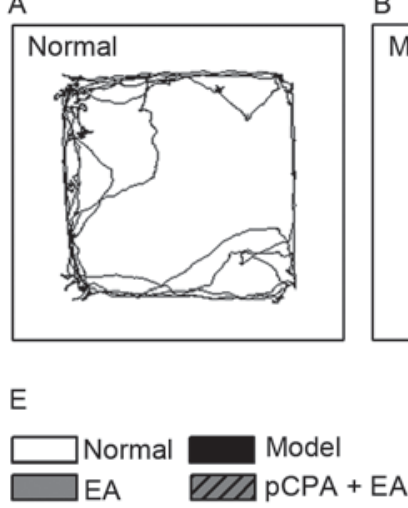

B

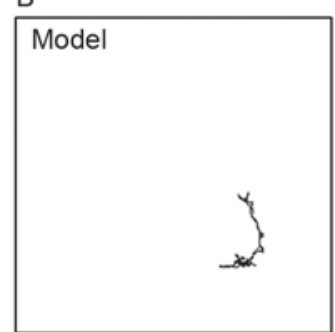

$\mathrm{F}$

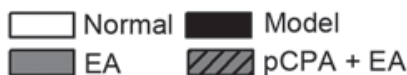

C

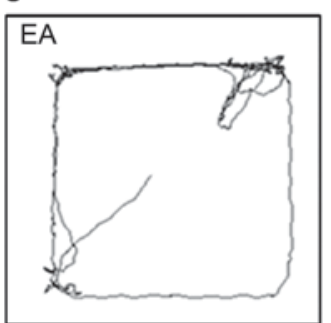

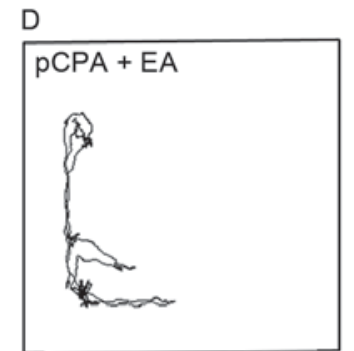

G
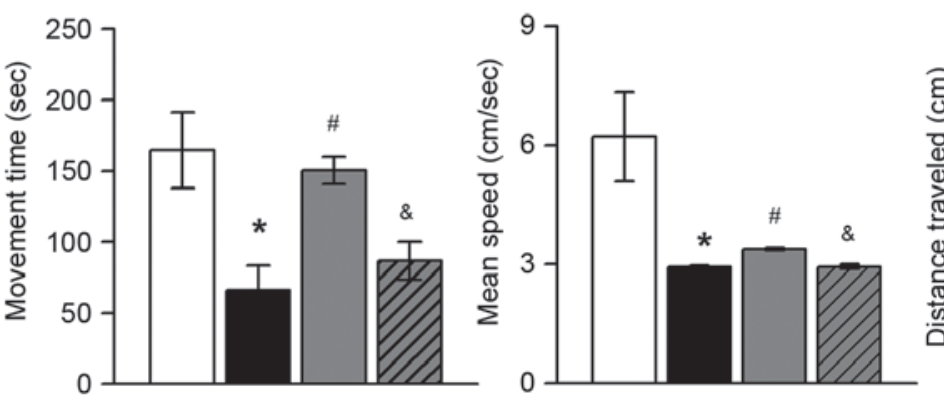
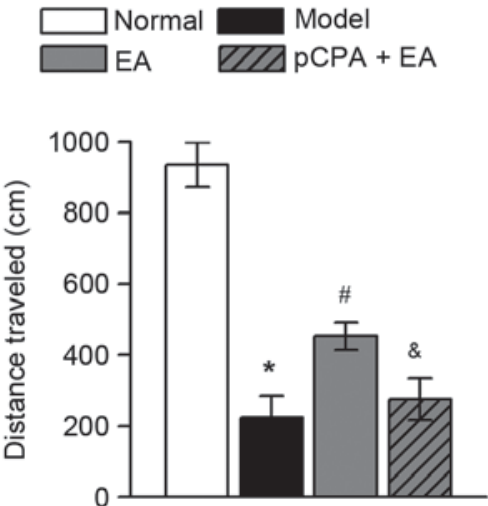

Figure 3. Effects of EA on depressive-like behavior in rats quantified using the OF test on day 5. Trajectories in OF test from (A) the normal group, (B) model group, (C) EA group and (D) pCPA + EA group. Effects of EA on (E) movement time, (F) mean speed and (G) distance traveled by rats with pain-depression dyad. Values are expressed as the mean \pm standard error of the mean $(n=10) .{ }^{*} \mathrm{P}<0.05$, vs. normal group; ${ }^{\#} \mathrm{P}<0.05$, vs. model group; ${ }^{\circledR} \mathrm{P}<0.05$, vs. EA group. EA, electroacupuncture; pCPA, para-chlorophenylalanine; OF, open field.

Chicago, IL, USA) was used for all data analysis. Statistical analysis was performed by one-way analysis of variance followed by a post-hoc test of the least significant differences for multiple comparisons. The data were analyzed using a two-tailed student's t-test for the behavior results, 5-HT levels and 5-HT cell expression levels. $\mathrm{P}<0.05$ was considered to indicate a statistically significant difference.

\section{Results}

EA increases the PWT in rats with pain-depression dyad via 5-HT. As is shown in Fig. 2, repeated injection of reserpine resulted in a significant decrease in the PWT of rats $(\mathrm{P}<0.05) .100-\mathrm{Hz}$ EA significantly increased the PWT in reserpine-injected rats $(\mathrm{P}<0.05$, vs. the model group). Injection of pCPA (i.c.v.) significantly restrained the effect of $100-\mathrm{Hz}$ EA on PWT ( $\mathrm{P}<0.05$, vs. the EA group).

EA reduces depressive-like behavior in rats with pain-depression dyad via 5-HT. The OF test was performed to observe depressive-like behavior in rats. Representative trajectories from the OF test for the normal group, model group, EA group and $\mathrm{pCPA}+\mathrm{EA}$ group are presented in Fig. 3A-D, respectively. Repeated injection of reserpine significantly reduced movement time, mean speed and distance traveled in rats (Fig. 3E-G, respectively; $\mathrm{P}<0.05$ ). Movement time, mean speed and distance traveled were significantly increased by $100-\mathrm{Hz}$ EA ( $\mathrm{P}<0.05$, vs. the model group). Injection of pCPA (i.c.v.) significantly restrained the effect of $100-\mathrm{Hz}$ EA on the three parameters mentioned above $(\mathrm{P}<0.05$, vs. the EA group).
EA reduces anxiety-like behavior in rats with pain-depression dyad via 5-HT. In certain rodent models of depression, anxiety-like responses are observed $(28,29)$. To determine whether anxiety-like behaviors accompany the pain-depression dyad, rats were subjected to the EZM test. Three-dimensional activities in the EZM test of animals from the normal group, model group, EA group, and pCPA + EA group are presented in Fig. 4A-D, respectively. Repeated injection of reserpine resulted in significant decreases in entries in the open arms, stretching time and distance traveled in rats (Fig. 4E-G, respectively; $\mathrm{P}<0.05$ ). 100-Hz EA significantly increased the number of entries in the open arms, stretching time and distance traveled in rats with pain-depression dyad $(\mathrm{P}<0.05$, vs. the model group). Although a declining trend of entries into the open arms existed in the pCPA + EA group, no significant difference was found between the EA group and the pCPA + EA group (Fig. 4E). Injection of pCPA (i.c.v.) significantly restrained the effects of $100-\mathrm{Hz}$ EA on stretching time and distance traveled (Fig. 4F and G, respectively; $\mathrm{P}<0.05$, vs. the EA group).

EA increases 5-HT in the DRN of rats with pain-depression dyad. HPLC-ECD was adopted to determine the effect of $100-\mathrm{Hz}$ EA on 5-HT levels in the DRN in rats with pain-depression dyad. Repeated injection of reserpine significantly decreased the 5-HT levels of DRN in rats (Fig. 5; $\mathrm{P}<0.05)$. 100-Hz EA significantly increased the 5-HT levels of DRN in reserpine-injected rats $(\mathrm{P}<0.05$, vs. the model group). The upregulation of 5-HT levels in DRN by $100-\mathrm{Hz}$ EA was completely abrogated by injection of pCPA (i.c.v.) in the $\mathrm{pCPA}+\mathrm{EA}$ group $(\mathrm{P}<0.05$, when compared to the EA group). 
A

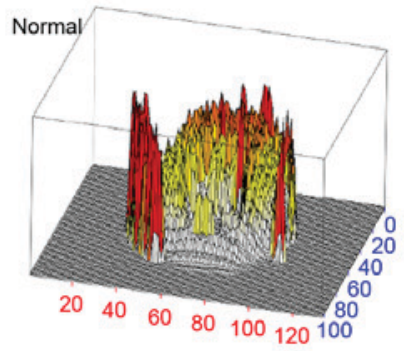

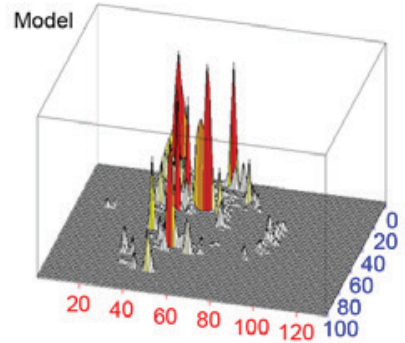

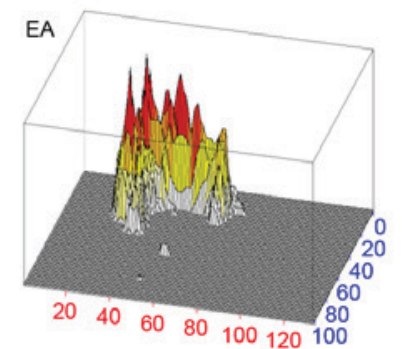

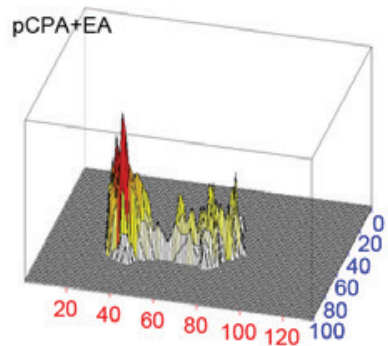
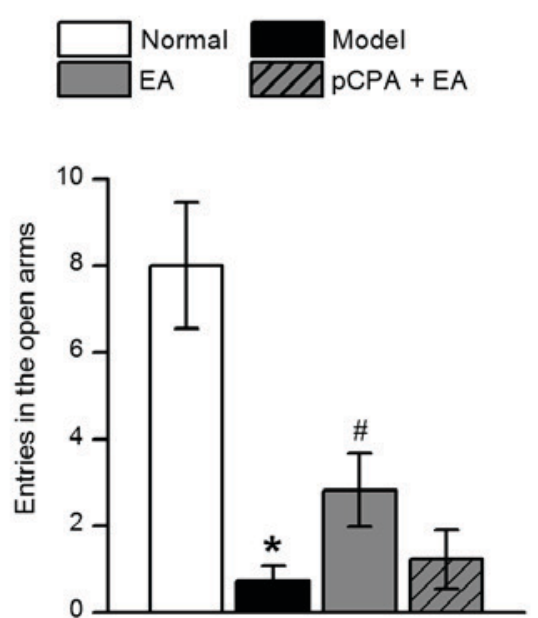
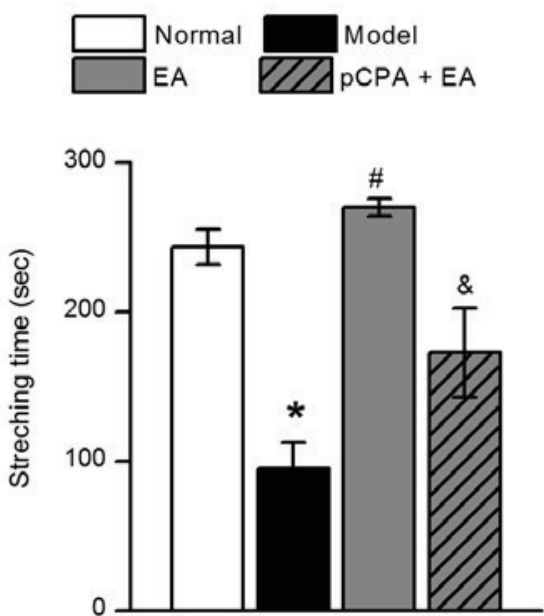

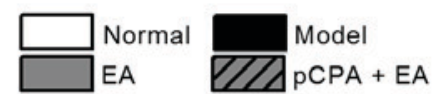

Figure 4. Effects of EA on the anxiety-like behavior in rats quantified by the EZM test on day 5. Three-dimensional activities in EZM test in (A) the normal group, (B) model group, (C) EA group and (D) pCPA + EA group. Effects of EA on (E) entries in the open arms, (F) stretching time and (G) distance traveled of rats with pain-depression dyad. Values are expressed as the mean \pm standard error of the mean $(\mathrm{n}=10)$. ${ }^{*} \mathrm{P}<0.05$, vs. normal group; ${ }^{*} \mathrm{P}<0.05$, vs. model group; ${ }^{\&} \mathrm{P}<0.05$, vs. EA group. EA, electroacupuncture; pCPA, para-chlorophenylalanine; EZM, elevated zero maze.

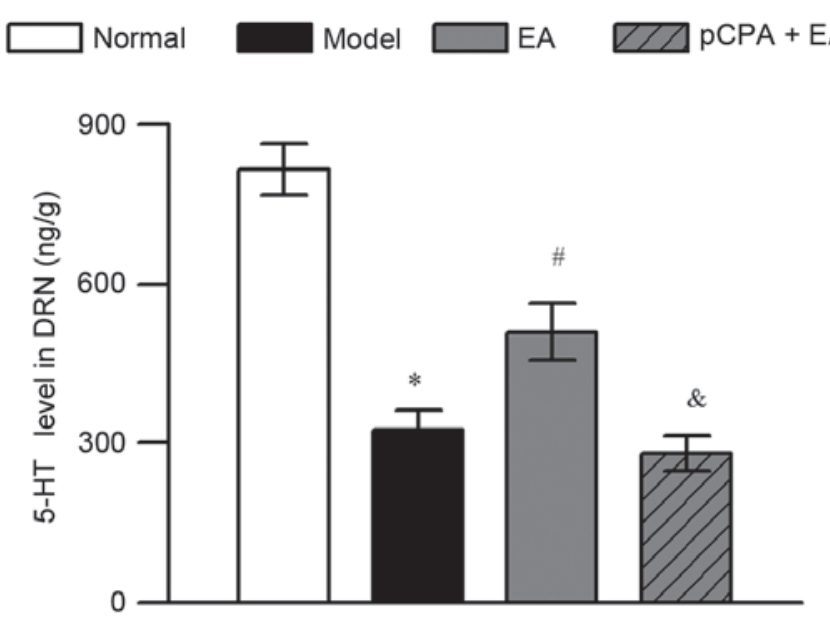

Figure 5. Effect of EA on 5-HT levels in the dorsal raphe nucleus of rats as determined by high-performance liquid chromatography circular electrochemical detection on day 5 . Values are expressed as the mean \pm standard error of the mean $(\mathrm{n}=7)$. ${ }^{*} \mathrm{P}<0.05$, vs. normal group; ${ }^{\#} \mathrm{P}<0.05$, vs. model group; ${ }^{\&} \mathrm{P}<0.05$, vs. EA group. EA, electroacupuncture; 5-HT, 5-hydroxytryptamine; pCPA, para-chlorophenylalanine.

EA enhances the number of 5-HT-immunoreactive cells in the DRN of rats with pain-depression dyad. Distribution of 5-HT-immunoreactive cells in the DRN of rats was investigated at bregma $-7.5,-8.0$ and $-8.5 \mathrm{~mm}$ (Fig. 6A-C, respectively). The abundance of 5-HT-immunoreactive cells in the DRN was higher at bregma $-8.00 \mathrm{~mm}$ than at bregma -7.50 and $-8.50 \mathrm{~mm}$. A significant decline of 5-HT-immunoreactive cells in the DRN compared with the normal group was found at bregma $-8.00 \mathrm{~mm}$ (Fig. 6D, $\mathrm{P}<0.05$ ), which was greater than that observed at the other points. Bregma $-8.0 \mathrm{~mm}$ was therefore determined to be the optimal location for examining the effect of $100-\mathrm{Hz}$ EA on 5-HT-immunoreactive cells in the DRN of rats with pain-depression dyad.

Representative images of 5-HT-immunoreactive cells in the DRN for the normal group, model group, EA group and pCPA + EA group are shown in Fig. 7A-D, respectively. As is shown in Fig. 7E, repeated injection of reserpine significantly reduced the number of 5-HT-immunoreactive cells in the DRN of rats $(\mathrm{P}<0.05)$. Of note, $100-\mathrm{Hz}$ EA significantly increased the number of 5-HT-immunoreactive cells in the DRN in reserpine-injected rats when compared to that in the model group $(\mathrm{P}<0.05)$. The upregulation of the number of 5 -HT-immunoreactive cells in DRN by $100-\mathrm{Hz}$ EA was totally abrogated by injection of pCPA (i.c.v.) in the pCPA + EA group $(\mathrm{P}<0.05$, when compared to the EA group).

\section{Discussion}

Pain-depression dyad is a complex illness with symptoms of pain overlapping with emotional disorders such as depression 

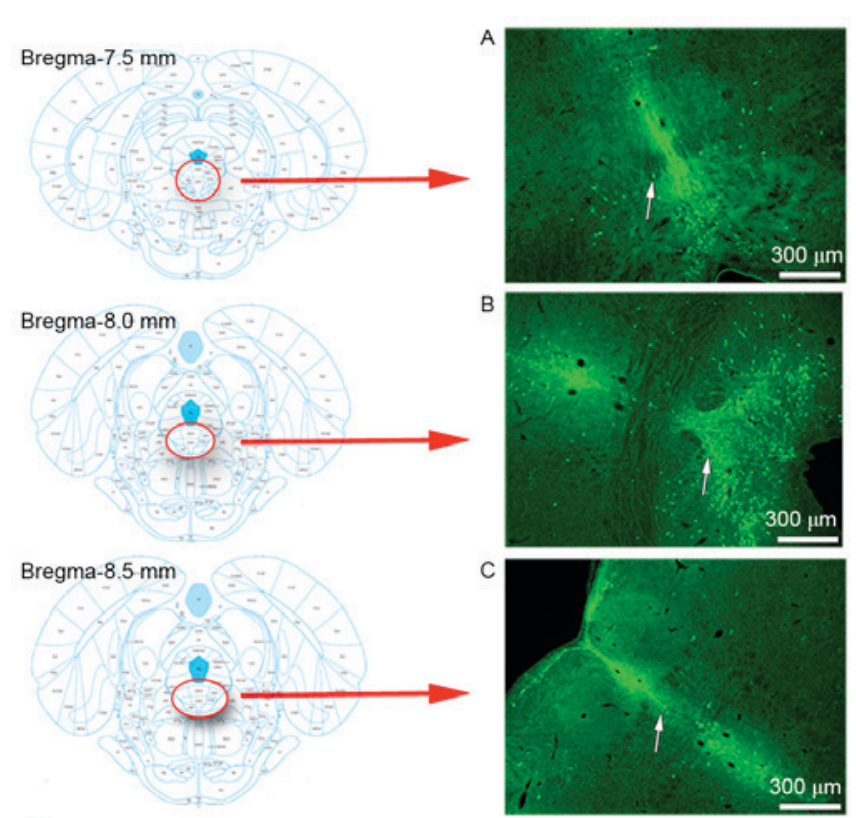

D

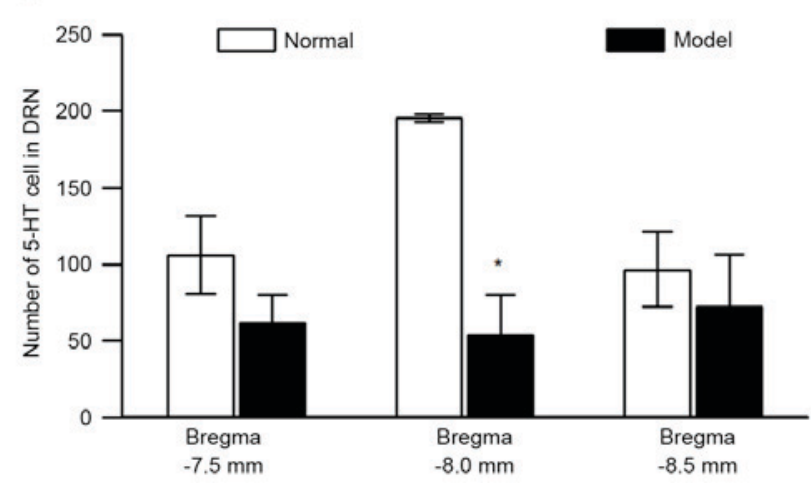

Figure 6. Distribution of 5-HT-immunoreactive cells in the DRN of rats detected by immunofluorescence on day 5. (A-C) Immunofluorescence of 5-HT expression in the DRN at bregma $-7.5,-8.0$ and $-8.5 \mathrm{~mm}$, respectively (scale bar, $300 \mu \mathrm{m}$ for all). White arrows indicate the 5-HT-immunoreactive cells. Insets (images from Paxinos and Watson, 1986) depict areas in which quantification was performed. (D) Quantification of 5-HT-immunoreactive cells in DRN. Values are expressed as the mean \pm standard error of the mean $(n=3)$. ${ }^{*} \mathrm{P}<0.05$, vs. normal group. DRN, dorsal raphe nucleus; 5 -HT, 5-hydroxytryptamine.

and anxiety (1). It has been reported that $52 \%$ patients with chronic pain suffer from depression, which is a costly health problem (30). While antidepressants and antiepileptics substantially reduce the symptoms, numerous patients cannot tolerate the side effects of their long-term administration. This dissatisfaction compelled researchers to explore complementary and alternative medicines $(1,6,9)$. A previous study by our group initially demonstrated that EA with high frequency but not low frequency effectively relieves pain-depression dyad (13). The present study found that 5-HT in the DRN was involved in the analgesic and anti-depressant effects of 100-Hz EA.

Several modeling methods are used for studying the mechanisms of the pain-depression dyad, including nerve injury (31), stress load $(32,33)$ and administration of reserpine (34), monosodium iodoacetate (35) and Freund's complete adjuvant (36). Reserpine-injected rats, an ideal model of pain-depression dyad, display hyperalgesia, allodynia and depressive behaviors accompanied with anxiety $(28,34,37)$.
A
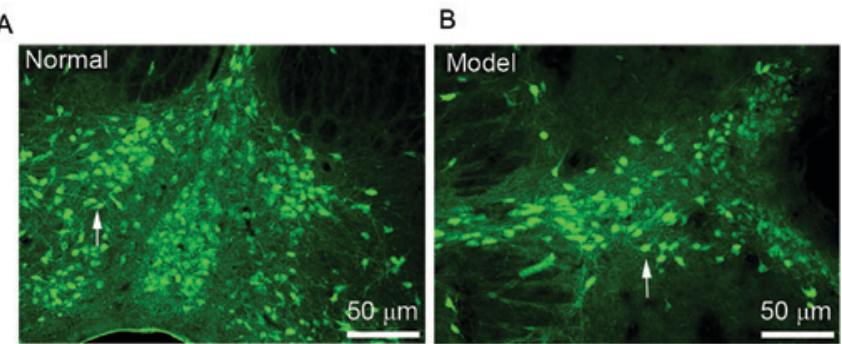

C

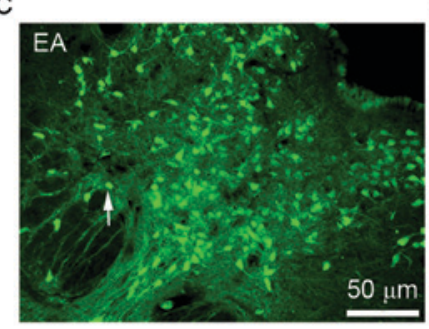

$\mathrm{D}$

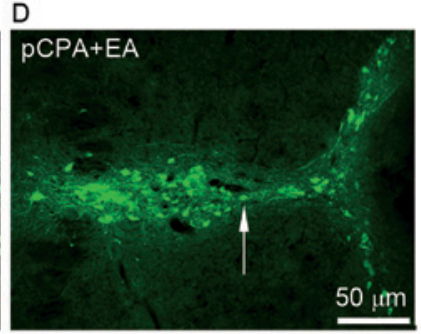

E

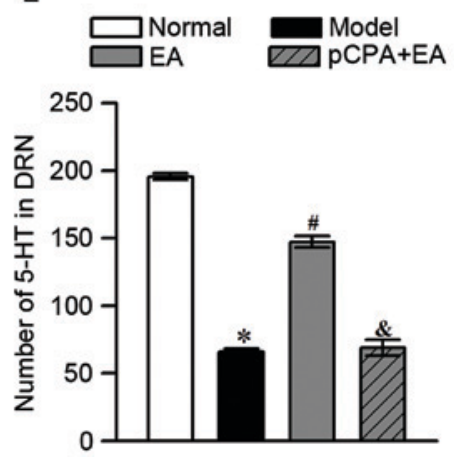

Figure 7. Effect of EA on 5-HT-immunoreactive cells in DRN assessed by immunofluorescence on day 5. (A-D) Immunofluorescence detection of 5-HT in the DRN of (A) the normal group, (B) model group, (C) EA group and (D) pCPA + EA group (scale bar, $50 \mu \mathrm{m}$ for all). White arrows indicate the 5-HT-immunoreactive cells. (E) Quantified amount of 5-HT-immunoreactive cells in DRN. Values are expressed as the mean \pm standard error of the mean $(\mathrm{n}=3)$. ${ }^{\text {}} \mathrm{P}<0.05$, vs. normal group; ${ }^{\#} \mathrm{P}<0.05$, vs. model group; ${ }^{\&} \mathrm{P}<0.05$, vs. EA group. DRN, dorsal raphe nucleus; EA, electroacupuncture; 5-HT, 5-hydroxytryptamine; pCPA, para-chlorophenylalanine.

The response is also similar to that of pain-depression patients in the clinic. This model was thus selected for use in the present study. The results showed that reserpine injection resulted in mechanical allodynia in rats. The forced swimming test, OF test and EZM test have been widely used in animal psychology for decades $(24,25,38)$. The forced swimming and $\mathrm{OF}$ tests are commonly adopted to evaluate depressive behaviors of animals (38). Considering that the forced swimming test can influence pain sensitivity in rodents $(39,40)$, the present study used the OF test to evaluate depressive behaviors in rats with reserpine-induced pain-depression dyad. As the reserpine-injected rats exhibited not only depressive-like but also anxiety-like behavior in the EZM test, it was indicated that the pain-depression dyad model was successfully established.

5-HT has an important role in the central nervous system in the descending control of pain or emotion (18,41-43). DRN is abundant of serotonergic neurons (42). The present study found that reserpine, a monoamine depletor, caused a decline of 5-HT in the DRN, which contributes to reserpine-induced allodynia and depressive behaviors in rats $(37,44)$. Acupuncture, particularly EA, is commonly used for pain or emotional 
problems $(7-9,19,45,46)$. The present and a previous study by our group showed that $100-\mathrm{Hz}$ EA effectively improved mechanical allodynia and depressive behaviors in rats caused by reserpine injection (13). However, the mechanism underlying the effects of EA on the pain-depression dyad has been rarely assessed. Studies have shown that high-frequency EA at the spinal cord is highly effective and exerts its effects by segmental inhibition at the spinal cord $(10,42,47,48)$ However, recent studies demonstrated that $100-\mathrm{Hz}$ EA improved Parkinson's disease (a typical brain-derived disease) and may exert its effects through the cerebrum $(49,50)$. In the present study, 100-Hz EA upregulated 5-HT DRN in rats with reserpine-induced pain-depression dyad. Furthermore, injection of pCPA (i.c.v.), an inhibitor of 5-HT resynthesis, suppressed the upregulation of 5-HT in the DRN by $100-\mathrm{Hz}$ EA and partially abrogated the analgesic and anti-depressive effects of $100-\mathrm{Hz}$ EA. These findings suggested that $100-\mathrm{Hz}$ EA improves reserpine-induced pain-depression dyad partially via 5-HT DRN. It may be assumed that high-frequency EA on body parts other than the spinal cord is also efficient.

In conclusion, the present study was the first, to the best of our knowledge, to demonstrate that 5-HT in the DRN participates in mediating the effects of $100 \mathrm{~Hz}$ EA on the pain-depression dyad. The present study provided a scientific basis for the value of high-frequency EA in treating supraspinal-originating diseases.

\section{Acknowledgements}

We acknowledge Mr. Sheng-Jian Zhuang and Mr. Jie Gong (postgraduate students) at Department of Neurobiology and Acupuncture Research, The Third Clinical Medical College, Zhejiang Chinese Medical University (Hangzhou, China) for their support with the behavioral testing of all of the animals. The present study was supported by the National Natural Science Foundation of China (nos. 81072855 and 81303039), the Zhejiang Provincial Natural Science Foundation of China (nos. Z2100979 and LY12H27015) and the Key Subject of State Administration of Traditional Chinese Medicine of China (Acupuncture and Moxibustion). This manuscript has been edited and proofread by Nature Publishing Group Language Editing.

\section{References}

1. Goldenberg DL: Pain/depression dyad: A key to a better understanding and treatment of functional somatic syndromes. Am J Med 123: 675-682, 2010.

2. Ohayon MM and Schatzberg AF: Using chronic pain to predict depressive morbidity in the general population. Arch Gen Psychiatry 60: 39-47, 2003.

3. Minami M: Neuronal mechanisms underlying pain-induced negative emotions. Brain nerve 64: 1241-1247, 2012 (In Japanese).

4. Wieser MJ, Gerdes AB, Reicherts P and Pauli P: Mutual influences of pain and emotional face processing. Front Psychol 5: 1160,2014

5. Keefe FJ, Lumley M, Anderson T, Lynch T, Studts JL and Carson KL: Pain and emotion: New research directions. J Clin Psychol 57: 587-607, 2001.

6. Bellato E, Marini E, Castoldi F, Barbasetti N, Mattei L, Bonasia DE and Blonna D: Fibromyalgia syndrome: Etiology, pathogenesis, diagnosis, and treatment. Pain Res Treat 2012: 426130, 2012.

7. Berman BM,Langevin HM, Witt CM and Dubner R: Acupuncture for chronic low back pain. N Engl J Med 363: 454-461, 2010.
8. Lin JG, Lo MW, Wen YR, Hsieh CL, Tsai SK and Sun WZ: The effect of high and low frequency electroacupuncture in pain after lower abdominal surgery. Pain 99: 509-514, 2002.

9. Weber A, Werneck L, Paiva E and Gans P: Effects of music in combination with vibration in acupuncture points on the treatment of fibromyalgia. J Altern Complement Med 21: 77-82, 2015.

10. Andersson SA and Holmgren E: Pain threshold effects of peripheral conditioning stimulation. In: Advances in Pain Research Therapy. Vol 1. Raven Press, New York, NY, pp761-768, 1976.

11. Youn JI, Sung KK, Song BK, Kim M and Lee S: Effects of electro-acupuncture therapy on post-stroke depression in patients with different degrees of motor function impairments: A pilot study. J Phys Ther Sci 25: 725-728, 2013.

12. Lumley MA, Cohen JL, Borszcz GS, Cano A, Radcliffe AM, Porter LS, Schubiner H and Keefe FJ: Pain and emotion: A biopsychosocial review of recent research. J Clin Psychol 67: 942-968, 2011.

13. Wu YY, Jiang YL, He XF, Zhao XY, Shao XM, Du JY and Fang JQ: Effects of electroacupuncture with dominant frequency at SP 6 and ST 36 based on meridian theory on pain-depression dyad in rats. Evid Based Complement Alternat Med 2015: 732845,2015

14. Ossipov MH, Dussor GO and Porreca F: Central modulation of pain. J Clin Invest 120: 3779-3787, 2010.

15. Freitas RL, Bassi GS, de Oliveira AM and Coimbra NC: Serotonergic neurotransmission in the dorsal raphe nucleus recruits in situ 5-HT $(2 \mathrm{~A} / 2 \mathrm{C})$ receptors to modulate the post-ictal antinociception. Exp Neurol 213: 410-418, 2008.

16. Lowry CA, Johnson PL, Hay-Schmidt A, Mikkelsen J and Shekhar A: Modulation of anxiety circuits by serotonergic systems. Stress 8: 233-246, 2005.

17. Lowry CA, Hollis JH, de Vries A, Pan B, Brunet LR, Hunt JR, Paton JF, van Kampen E, Knight DM, Evans AK, et al: Identification of an immune-responsive mesolimbocortical serotonergic system: Potential role in regulation of emotional behavior. Neuroscience 146: 756-772, 2007.

18. Xu SF: Pain and analgesia. In: Neurology. Wei L (ed.) Vol 1. 2nd edition. Fudan University Press, Shanghai, pp330-349, 1999 (In Chinese).

19. Yano T, Kato B, Fukuda F, Shinbara H, Yoshimoto K, Ozaki A and Kuriyama K: Alterations in the function of cerebral dopaminergic and serotonergic systems following electroacupuncture and moxibustion applications: Possible correlates with their antistress and psychosomatic actions. Neurochem Res 29: 283-293, 2004.

20. Bertholomey ML, Henderson AN, Badia-Elder NE and Stewart RB: Neuropeptide Y (NPY)-induced reductions in alcohol intake during continuous access and following alcohol deprivation are not altered by restraint stress in alcohol-preferring $(\mathrm{P})$ rats. Pharmacol Biochem Behav 97: 453-461, 2011.

21. Paxinos $G$ and Watson $C$ : The rat brain in stereotaxic coordinates. Academic Press, San Diego, CA, 1986.

22. Thibault K, Calvino B, Rivals I, Marchand F, Dubacq S, McMahon SB and Pezet S: Molecular mechanisms underlying the enhanced analgesic effect of oxycodone compared to morphine in chemotherapy-induced neuropathic pain. PLoS One 9: e91297, 2014.

23. Thibault K, Elisabeth B, Sophie D, Claude FZ, Bernard R and Bernard C: Antinociceptive and anti-allodynic effects of oral PL37, a complete inhibitor of enkephalin-catabolizing enzymes, in a rat model of peripheral neuropathic pain induced by vincristine. Eur J Pharmacol 600: 71-77, 2008.

24. Shepherd JK, Grewal SS, Fletcher A, Bill DJ and Dourish CT: Behavioural and pharmacological characterisation of the elevated 'zero-maze' as an animal model of anxiety. Psychopharmacology (Berl) 116: 56-64, 1994.

25. Gould TD, Dao DT and Kovacsics CE: The open field test. In: Mood and anxiety related phenotypes in mice. Gould TD (ed). Humana Press, Totowa, NJ, pp1-20, 2009.

26. Singer S, Rossi S, Verzosa S, Hashim A, Lonow R, Cooper T, Sershen H and Lajtha A: Nicotine-induced changes in neurotransmitter levels in brain areas associated with cognitive function. Neurochem Res 29: 1779-1792, 2004.

27. Pani AK, Jiao Y, Sample KJ and Smeyne RJ: Neurochemical measurement of adenosine in discrete brain regions of five strains of inbred mice. PLoS One 9: e92422, 2014.

28. Fukui M, Rodriguiz RM, Zhou J, Jiang SX, Phillips LE, Caron MG and Wetsel WC: Vmat2 heterozygous mutant mice display a depressive-like phenotype. J Neurosci 27: 10520-10529, 2007.

29. De Vry J, Vanmierlo T, Martínez-Martínez P, Losen M, Temel Y, Boere J, Kenis G, Steckler T, Steinbusch HW, De Baets M and Prickaerts J: TrkB in the hippocampus and nucleus accumbens differentially modulates depression-like behavior in mice. Behav Brain Res 296: 15-25, 2016. 
30. Bair MJ, Robinson RL, Katon W and Kroenke K: Depression and pain comorbidity: A literature review. Arch Intern Med 163: 2433-2445, 2003.

31. Alba-Delgado C, Llorca-Torralba M, Horrillo I, Ortega JE, Mico JA, Sánchez-Blázquez P, Meana JJ and Berrocoso E: Chronic pain leads to concomitant noradrenergic impairment and mood disorders. Biol Psychiatry 73: 54-62, 2013.

32. Rojas-Corrales MO, Berrocoso E, Gibert-Rahola J and Micó JA: Antidepressant-like effects of tramadol and other central analgesics with activity on monoamines reuptake, in helpless rats. Life Sci 72: 143-152, 2002.

33. Wang N, Shi M, Wang JY and Luo F: Brain-network mechanisms underlying the divergent effects of depression on spontaneous versus evoked pain in rats: A multiple single-unit study. Exp Neurol 250: 165-175, 2013.

34. Taguchi T, Katanosaka K, Yasui M, Hayashi K, Yamashita M, Wakatsuki K, Kiyama H, Yamanaka A and Mizumura K: Peripheral and spinal mechanisms of nociception in a rat reserpine-induced pain model. Pain 156: 415-427, 2015.

35. Stevenson GW, Mercer H, Cormier J, Dunbar C, Benoit L, Adams C, Jezierski J, Luginbuhl A and Bilsky EJ: Monosodium iodoacetate-induced osteoarthritis produces pain-depressed wheel running in rats: Implications for preclinical behavioral assessment of chronic pain. Pharmacol Biochem Behav 98: 35-42, 2011.

36. Stein C, Millan MJ and Herz A: Unilateral inflammation of the hindpaw in rats as a model of prolonged noxious stimulation: Alterations in behavior and nociceptive thresholds. Pharmacol Biochem Behav 31: 445-451, 1988.

37. Arora V, Kuhad A, Tiwari V and Chopra K: Curcumin ameliorates reserpine-induced pain-depression dyad: Behavioural, biochemical, neurochemical and molecular evidences. Psychoneuroendocrinology 36: 1570-1581, 2011.

38. Chang CY, Guo HR, Tsai WC, Yang KL, Lin LC, Cheng TJ and Chuu JJ: Subchronic arsenic exposure induces anxiety-like behaviors in normal mice and enhances depression-like behaviors in the chemically induced mouse model of depression. Biomed Res Int 2015: 159015, 2015.

39. Ibironke GF and Rasal KS: Forced swimming stress-related hypoalgesia: Nondependence on the histaminergic mechanisms. Neurophysiology 45: 340-343, 2013.
40. Łapo IB, Konarzewski M and Sadowski B: Analgesia induced by swim stress: Interaction between analgesic and thermoregulatory mechanisms. Pflugers Arch 446: 463-469, 2003.

41. McDevitta RA and Neumaier JF: Regulation of dorsal raphe nucleus function by serotonin autoreceptors: A behavioral perspective. J Chem Neuroanat 41: 234-246, 2011.

42. Han JS: 5-hydroxytryptamine. In: Neuroscience. Han ZG and Liu Y (eds.). Vol 1.3rd edition. Peking University Medical Press, Beijing, pp382-351, 2009 (In Chinese)

43. Hale MW, Dady KF, Evans AK and Lowry CA: Evidence for in vivo thermosensitivity of serotonergic neurons in the rat dorsal raphe nucleus and raphe pallidus nucleus implicated in thermoregulatory cooling. Exp Neurol 227: 264-278, 2011.

44. Nagakura $\mathrm{Y}, \mathrm{Oe} \mathrm{T}$, Aoki $\mathrm{T}$ and Matsuoka N: Biogenic amine depletion causes chronic muscular pain and tactile allodynia accompanied by depression: A putative animal model of fibromyalgia. Pain 146: 26-33, 2009.

45. Sniezek DP and Siddiqui IJ: Acupuncture for treating anxiety and depression in women: A clinical systematic review. Med Acupunct 25: 164-172, 2013.

46. Park H, Yoo D, Kwon S, Yoo TW, Park HJ, Hahm DH, Lee H and Kim ST: Acupuncture stimulation at HT7 alleviates depression-induced behavioral changes via regulation of the serotonin system in the prefrontal cortex of maternally-separated rat pups. J Physiol Sci 62: 351-357, 2012.

47. Ogata A, Sugenoya J, Nishimura $\mathrm{N}$ and Matsumoto T: Low and high frequency acupuncture stimulation inhibits mental stress-induced sweating in humans via different mechanisms. Auton Neurosci 118: 93-101, 2005.

48. Fung SJ and Chan SH: Primary afferent depolarization evoked by electroacupuncture in the lumbar cord of the cat. Exp Neurol 52: 168-176, 1976.

49. Du J, Sun ZL, Jia J, Wang X and Wang XM: High-frequency electro-acupuncture stimulation modulates intracerebral $\gamma$-aminobutyric acid content in rat model of Parkinson's disease. Sheng Li Xue Bao 63: 305-310, 2011.

50. Rui G, Guangjian Z, Yong W, Jie F, Yanchao C, Xi J and Fen L: High frequency electro-acupuncture enhances striatum DAT and D1 receptor expression, but decreases D2 receptor level in 6-OHDA lesioned rats. Behav Brain Res 237: 263-269, 2013. 